\title{
Carbon hydrangeas with typical ionic liquid matched pores for advanced supercapacitors
}

Ziyang Song, ${ }^{\text {a Hui Duan, }}{ }^{\text {a }}$ Ling Miao, ${ }^{\mathrm{a}}$ Laurent Ruhlmann, ${ }^{\mathrm{b}}$ Yaokang Lv, ${ }^{\text {be }}$ Zhiwei Wang, ${ }^{\mathrm{c}}$ Wei Xiong, ${ }^{\mathrm{d}}$ Dazhang Zhu, ${ }^{\mathrm{a}}$ Liangchun $\mathrm{Li},{ }^{\mathrm{a}}$ Lihua Gan, ${ }^{\mathrm{a} *}$ Mingxian Liu ${ }^{\mathrm{ac} *}$

${ }^{a}$ Shanghai Key Lab of Chemical Assessment and Sustainability, School of Chemical Science and Engineering, Tongji University, Shanghai, 200092, P. R. China.

${ }^{b}$ Institut de Chimie (UMR au CNRS n 7177 ), Université de Strasbourg, 4, rue Blaise Pascal CS 90032, F-67081 Strasbourg Cedex

${ }^{c}$ State Key Laboratory of Pollution Control and Resources Reuse, Shanghai Institute of Pollution Control and Ecological Security, School of Environmental Science and Engineering, Tongji University, Shanghai 200092, P. R. China

${ }^{d}$ Key Laboratory for Green Chemical Process of Ministry of Education, School of Chemistry and Environmental Engineering, Wuhan Institute of Technology, 693 Xiongchun Road, Wuhan 430073, P. R. China.

${ }^{e}$ College of Chemical Engineering, Zhejiang University of Technology, Hangzhou 310014, P. R. China.

\section{*Corresponding Authors}

E-mail: ganlh@tongji.edu.cn, liumx@,tongji.edu.cn 


\begin{abstract}
Pore-ion size matching between carbon electrodes and electrolytes is crucial for superior energy storage. However, it remains a great challenge to engineer carbons with perfectly compatible pore dimension for desired electrolytes. Herein we design a simple synthetic route to carbon hydrangeas integrated with unique geometry, high surface areas, N/O doping, and more importantly, well-controlled pore structure. The narrow primary subnanopores of $0.80 \mathrm{~nm}$ are exactly matched the cation sizes $\left(\mathrm{EMIM}^{+}, 0.76 \mathrm{~nm}\right)$ of ionic liquid electrolytes, 1-ethyl-3methylimidazolium bis(trifluoromethylsulfonyl)imide (EMIM.TFSI) and 1-ethyl-3methylimidazolium tetrafluoroborate $(\mathrm{EMIM} \mathrm{BF} 4)$. Besides, the secondary pores of $0.50 \mathrm{~nm}$ are accessible for the smaller $\mathrm{BF}_{4}{ }^{-}$anions $(0.48 \mathrm{~nm})$ relative to $\mathrm{TFSI}^{-}(0.79 \mathrm{~nm})$, giving enhanced ion diffusion/adsorption kinetics. The fabricated solid-state supercapacitor based on EMIM.BF 4 gel electrolyte achieves a record energy density of $101.2 \mathrm{Wh} \mathrm{kg}^{-1}$, with $29.2 \%$ enhancement against the use of EMIM.TFSI. This study presents a break-through to develop functionalized carbons with narrow and perfectly ion-comparable pore architecture, moving toward advanced energy storage systems.
\end{abstract}




\section{Introduction}

As one of the most advanced energy storage devices, carbon-based supercapacitors (CSs) have attractedconsiderable attention because of outstanding cycle lifetime, competitive power density and reliable operation safety. ${ }^{1-5}$ CSs store energy through potential-driven accumulation of electrolyte ions to counterbalance the surface charge of porous carbon electrodes, identified as electric double layer formation. ${ }^{6-10}$ However, only limited energy densities are obtained for such systems, which are about an order of magnitude lower than those of Li-ion batteries. ${ }^{11,12}$ Consequently, unlocking the energy potential of CSs without sacrificing the power output is highly required, to extend their utilization into high-energy applications. ${ }^{13-17}$ To achieve this goal, considerable efforts have been devoted to design diverse carbon electrode materials with tunable geometry and rational pore size distribution for efficient charge/ion transfer. Doped heteroatoms have been also incorporated for enhanced surface wettability and additional pseudocapacitance, usually associated with the use of high-voltage electrolytes such as ionic liquids (ILs). ${ }^{18-21}$

The capacity of a CSs, or more intuitively, the number of charged ions adsorbed, is closely relevant to the pore architecture of the electroactive materials and the ion sizes of the used electrolyte. ${ }^{22}$ Gogotsi's group firstly reported the anomalous capacitance promotion in the case of nanocarbons with pore sizes smaller than $1 \mathrm{~nm}$, defying long-held axiom about inaccessibility of small pores to solvated ions. ${ }^{23}$ The abnormal phenomenon is ascribed to a desolvation of the electrolyte ions entering subnanopores, resulting in a sieving effect at pore sizes below the size of the solvated ions. ${ }^{23}$ This result gives a better understanding of ionic transport/accumulation into porous media for realizing efficient charge storage in CSs. Further experimental and theoretical studies have established that the pore size of an electrode tailored for achieving the maximal capacitance should be very approximate to the ion dimension of a given electrolyte. ${ }^{13,24,25}$ If the 
pore size is too small, the confined pores are ion-inaccessible. If the pore size is too large, there will be a wider space between ions and pore wall, both leading to a notable decrease of the capacitance value. This means, ingenious regulation and optimization of the pore size to perfectly match the dimensionality of desired electrolyte ions, is a key tactic to help improve ion transport/adsorption dynamics. However, up till now, it is a great challenging to govern the pores of carbons in a the subnanometer region, especially with an electrolyte ion-adapted level.

Herein we report the development of heteroatom-doped hydrangea-like porous carbons (HHPCs) with ion-comparable pore sizes for advanced CSs, using IL/gel-polymer electrolytes. HHPCs are designed based on a facile polymerization of benzoquinone and benzidine, which hold unique geometry and large surface area with improved surface wettability due to high-level N/O doping. Two ILs, 1-ethyl-3-methylimidazolium bis(trifluoromethylsulfonyl)imide (EMIM.TFSI) and 1ethyl-3-methylimidazolium tetrafluoroborate $\left(\mathrm{EMIM} \mathrm{BF}_{4}\right)$ with identical cation $\left(\mathrm{EMIM}^{+}, 0.76 \mathrm{~nm}\right)$ but with different anions (TFSI ${ }^{-}, 0.79 \mathrm{~nm} ; \mathrm{BF}_{4}^{-}, 0.48 \mathrm{~nm}$ ) are used as electrolytes to investigate the electrochemical energy storage capability. HHPCs exhibit dominant $0.80 \mathrm{~nm}$ and subordinate $0.50 \mathrm{~nm}$ pores. The dominant pore is perfectly comparable to the cation dimension of both ILs $\left(\mathrm{EMIM}^{+}\right)$while the secondary pores is well-matched to the anion size of tetrafluoroborate, $\mathrm{BF}_{4}{ }^{-}$. As a result, HHPCs-based solid-state symmetric supercapacitor using EMIM.BF 4 gel electrolyte delivers an extraordinary energy density of $101.2 \mathrm{Wh} \mathrm{kg}^{-1}$ against the substitute of EMIM.TFSI (78.3 $\mathrm{Wh} \mathrm{kg}^{-1}$ ). This study opens up a new opportunity for employing pore-ion size matching criterion in the building of high-efficient energy storage devices.

\section{Experimental}




\subsection{Synthesis of HHPCs}

Benzoquinone (3.24 g, $0.03 \mathrm{~mol}$ ) was added into the mixture of benzidine (1.84 g, $0.01 \mathrm{~mol})$ and ethanol $(100 \mathrm{~mL})$ at $70^{\circ} \mathrm{C}$, followed by stirring for $2 \mathrm{~h}$ to obtain a polymer. After filtrating, washing and drying, the polymer was mixed with $\mathrm{KOH}(1: 1, \mathrm{w} / \mathrm{w})$, and then carbonized/activated at $650-850{ }^{\circ} \mathrm{C}\left(3^{\circ} \mathrm{C} \mathrm{min}^{-1}\right)$ for 2 hours to fabricate HHPCs (labelled as $\mathrm{HHPC}_{x}$ where $x$ denotes the carbonization temperature).

\subsection{Characterization}

Thermogravimetric analysis was tested using a synchronous thermal analyzer (NETZSCH STA409 PC) at a heating rate $10{ }^{\circ} \mathrm{C} \mathrm{min}^{-1}$ under an $\mathrm{N}_{2}$ atmosphere. Fourier transform infrared spectroscopy (FT-IR) spectrum was recorded on a Thermo Nicolet NEXUS spectrometer. XRD patterns were characterized on a Bruker D8 diffractometer equipped with $\mathrm{Cu}$ radiation. The sample morphology was observed by a scanning electron microscopy (SEM, Hitachi S-4800). Scanning transmission electron microscopy (STEM) and corresponding elemental mapping characterization were performed on a JEM-F200 instrument equipped with an energy diffraction system. X-ray photoelectron spectrometer (XPS, AXIS Ultra DLD) was utilized to analyze the surface chemical composition. $\mathrm{N}_{2}$ adsorption/desorption measurement was collected at $-196{ }^{\circ} \mathrm{C}$ using a micromeritics apparatus (ASAP 2460). The surface area and pore size distribution were investigated by the Brunauer-Emmett-Teller method and nonlocal density functional theory.

\subsection{Assembly of supercapacitors}

The working electrode was prepared by pressing a slurry of HHPCs (80 wt $\%)$, 
polytetrafluoroethylene $(10 \mathrm{wt} \%)$, and graphite $(10 \mathrm{wt} \%)$ mixture onto a piece of stainless steel mesh and then dried at $80^{\circ} \mathrm{C}$. The mass loading of active substance on each working electrode is about $10 \mathrm{mg} \mathrm{cm}$. To obtain IL/gel-polymer electrolytes, $5 \mathrm{~g}$ of polyvinylidenefluoride-cohexafluoropropylene (PVDF-HFP) and a proper amount of EMIM.BF 4 or EMIM.TFSI was dissolved in $50 \mathrm{~mL}$ acetone, and then the mixture was magnetically stirred at $50{ }^{\circ} \mathrm{C}$ to obtain a homogeneous and transparent solution. The solution was put in a glass petri dish for acetone evaporation and dried in vacuum for 12 hours to form EMIM.BF $4 /$ PVDF-HFP and EMIMTFSI/PVDF-HFP gel-polymer electrolytes. Solid-state supercapacitors were assembled in a glovebox by stacking two identical working electrodes with an EMIM.TFSI or EMIM.BF4 gelpolymer electrolyte in the middle. To obtain a flexible device, the configuration was sealed by adhesive tape and compressed with appropriate pressure to get good interfacial contact between electrode/electrolyte.

\subsection{Electrochemical measurement}

All electrochemical measurements including cyclic voltammetry (CV), galvanostatic chargedischarge (GCD), and electrochemical impedance spectroscopy (EIS) were conducted with a CHI660E electrochemical workstation. The gravimetric capacitances $\left(C_{\mathrm{s}}, \mathrm{F} \mathrm{g}^{-1}\right)$ of a single electrode were calculated from GCD profiles according to form (1):

$$
C_{\mathrm{s}}\left(\mathrm{F} \mathrm{g}^{-1}\right)=\frac{4 \times I \times \Delta t}{m \times \Delta V}
$$

where $I(\mathrm{~A}), \Delta t(\mathrm{~s}), m(\mathrm{~g})$ and $\Delta V(\mathrm{~V})$ represents charge current, discharge time, the total mass of active material on two electrodes and the voltage window, respectively.

The gravimetric energy $(E)$ and power densities $(P)$ of the devices are denoted as follows: 


$$
\begin{aligned}
E\left(\mathrm{Wh} \mathrm{kg}^{-1}\right) & =\frac{1}{7.2 \times 4} C_{\mathrm{s}} \Delta V^{2} \\
E_{\mathrm{V}}\left(\mathrm{Wh} \mathrm{L}^{-1}\right) & =\rho \times E=\left(V_{\mathrm{t}}+1 / \rho_{\text {carbon }}\right)^{-1} \times E \\
P\left(\mathrm{~W} \mathrm{~kg}^{-1}\right) & =\frac{E}{\Delta t} \times 3600 \\
P_{\mathrm{v}}\left(\mathrm{W} \mathrm{L} \mathrm{L}^{-1}\right) & =\rho \times P
\end{aligned}
$$

where $E_{\mathrm{v}}$ and $P_{\mathrm{v}}$ represents the volumetric energy and power density of the symmetrical devices, respectively, and $V_{\mathrm{t}}$ denotes the total pore volume of HHPCs, and $\rho_{\text {carbon }}$ is the true density of carbon $\left(2 \mathrm{~g} \mathrm{~cm}^{-3}\right)$

\section{Results and discussion}

HHPCs was synthesized based on a Michael addition reaction between benzidine (electron-pair donicity) and benzoquinone (electron-drawing capability) with a mild condition. This strategy avoids any harsh and/or complicated procedures (e.g., template synthesis, high-temperature hydrothermal treatment, self-assembly, chemical vapor deposition), time-consuming process or multistep manipulation, which provides a highly efficient and competitive route for engineering functionalized porous carbons. Fig. S1 displays FT-IR spectrum of the benzidine-benzoquinone polymer, in which sharp peaks at 1564 and $1483 \mathrm{~cm}^{-1}$ can be assigned to the stretching vibration

of $\mathrm{C}=\mathrm{O}$ and $\mathrm{C}=\mathrm{C}$ groups. ${ }^{26,27}$ The characteristic absorption peaks centered at 1284 and $813 \mathrm{~cm}^{-1}$ are attributed to the stretching vibration of $\mathrm{C}-\mathrm{N}$ groups and the blending vibration of $-\mathrm{NH}-$ groups, respectively. ${ }^{28}$ Besides, the peak at $1178 \mathrm{~cm}^{-1}$ is ascribed to the stretching vibration of phenolic - OH groups in the formed hydroquinone resulted from the partial oxidation of benzoquinone. XRD profiles (Fig. S2) reveal two signals at about 26 and $43^{\circ}$ indexed to the (002) and (100) planes, suggesting an amorphous carbon framework. ${ }^{29}$ HHPCs has a high carbon yield of $71 \%$ at $750{ }^{\circ} \mathrm{C}$ 
(Fig. S3), and show hydrangea-like micron-scale carbon spheres with refined surface structure composing of enormous tunnels and furrows $($ Fig. $1 \mathrm{a}-\mathrm{c})$. Such a unique architecture allows rapid ion diffusion kinetics for full access of electrolyte ions into the adsorption sites. The elemental mapping images corresponding to STEM image (Fig. 1d-g) clearly displays the presence of C, N and $\mathrm{O}$ atoms and a homogeneous distribution in the carbon skeleton.
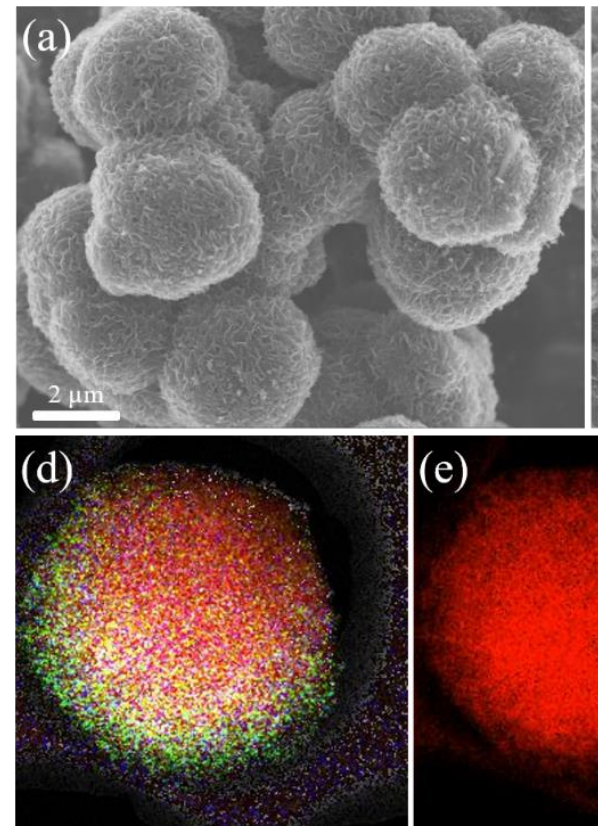
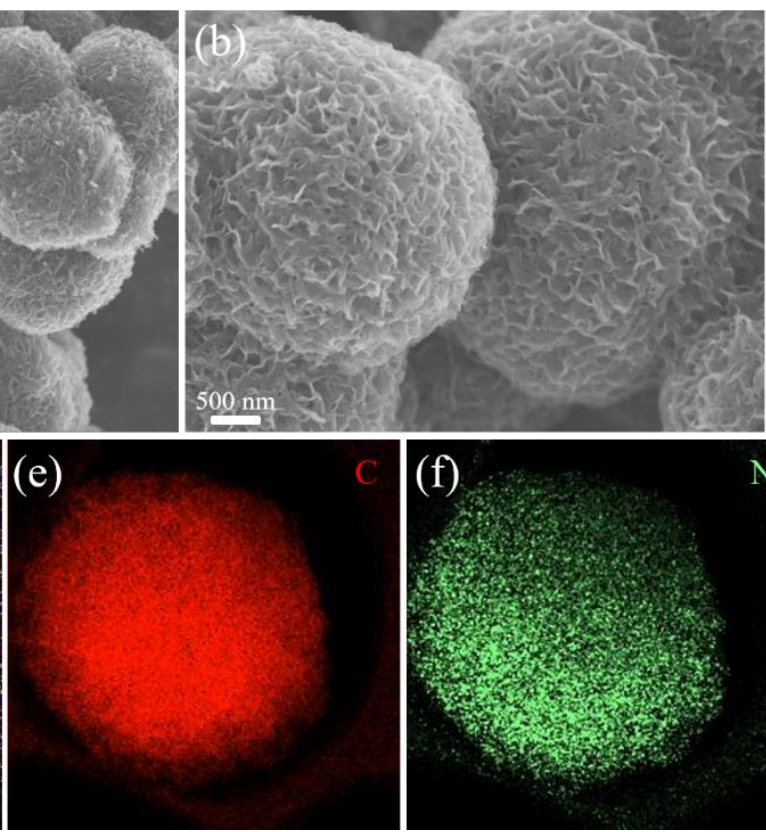

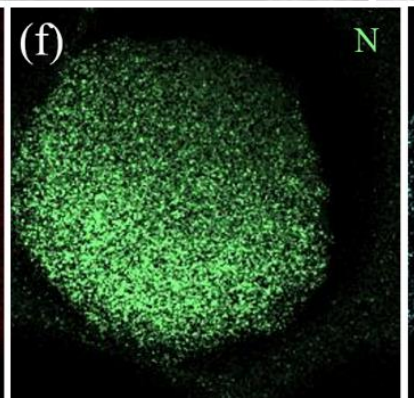

Fig. 1. (a, b) SEM images, (c) a TEM image, (d) energy-dispersive X-ray images and corresponding

(e) $\mathrm{C},(\mathrm{f}) \mathrm{N},(\mathrm{g}) \mathrm{O}$ element mapping images of $\mathrm{HHPC}_{750}$.

XPS survey spectra (Fig. 2a) of HHPCs demonstrate three signals at 285.3, 399.6 and $532.4 \mathrm{eV}$ corresponding to $\mathrm{C} 1 \mathrm{~s}, \mathrm{~N}$ 1s and $\mathrm{O}$ 1s peaks, respectively. With elevating carbonization temperature from 650 to $850{ }^{\circ} \mathrm{C}$, the $\mathrm{C}$ content increases from 76.01 to $87.89 \mathrm{wt} \%$, with the $\mathrm{N}$ content decreasing from 8.70 to 2.84 wt.\% (Table 1). The $\mathrm{O}$ content declines from 15.28 to $9.21 \mathrm{wt} . \%$ and remains unchanged at $850{ }^{\circ} \mathrm{C}$ owing to the high-bond-energy $\mathrm{C}=\mathrm{O}$ double bond $\left(799 \mathrm{~kJ} \mathrm{~mol}^{-1}\right)$ relative to a $\mathrm{C}-\mathrm{N}$ single bond $\left(305 \mathrm{~kJ} \mathrm{~mol}^{-1}\right)$. The highly stable $\mathrm{C}=\mathrm{O}$ covalent bond can effectively resist the bond breaking and the release of $\mathrm{O}$ species during carbonization. ${ }^{28,30}$ High-resolution $\mathrm{C}$ 1s spectra (Fig. S4) can be deconvoluted into four peaks positioned at about 284.4, 285.4, 286.7 and 288.5 
$\mathrm{eV}$, assigned to $\mathrm{C}=\mathrm{C} / \mathrm{C}-\mathrm{C}, \mathrm{C}-\mathrm{N}, \mathrm{C}-\mathrm{O}$ and $\mathrm{C}=\mathrm{O}$ components, respectively. ${ }^{31,32} \mathrm{~N}$ 1s species (Fig. 2c) include pyridine pyridinic $\mathrm{N}(\mathrm{N}-6,398.2 \mathrm{eV})$, pyrrolic $\mathrm{N}(\mathrm{N}-5,399.6 \mathrm{eV})$, quaternary $\mathrm{N}(\mathrm{N}-\mathrm{Q}$, 400.6 eV), as well as pyridine $\mathrm{N}$-oxide $(\mathrm{N}-\mathrm{X}, 402.9 \mathrm{eV}) .{ }^{33,34}$ The first three nitrogen species are proposed to provide stable pseudocapacitance in acid and base electrolytes through Faradic redox reactions, while the last one is beneficial to reduce effectively the electronic transfer resistance. O 1s spectra (Fig. 2d) can be fitted to three peaks at 530.9, 532.5, and $533.6 \mathrm{eV}$, reflecting the $\mathrm{C}=\mathrm{O}$, $\mathrm{C}-\mathrm{OH} / \mathrm{C}-\mathrm{O}-\mathrm{C}$, and $\mathrm{COO}-$ functionalities. ${ }^{35}$
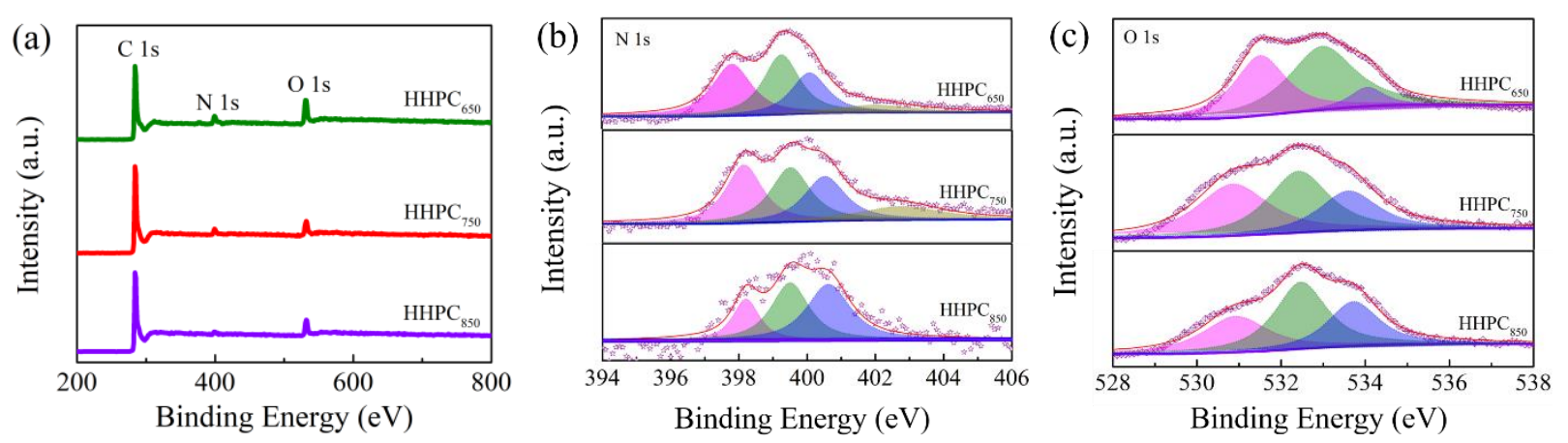

Fig. 2. (a) XPS survey spectra and high-resolution (b) N 1s and (c) O 1s spectra of HHPCs.

As depicted in Fig. 3a, HHPCs demonstrate combined type-I and IV isotherms which consist of steep adsorption regions in the low-pressure $\left(P / P_{0}<0.1\right)$, and well-defined plateaus with distinctive hysteresis loops $\left(P / P_{0}>0.45\right)$, implying the presence of micro- and mesopores. ${ }^{36,37}$ HHPCs exhibit micropore sizes concentrated at $\sim 0.50,0.80$ and $1.26 \mathrm{~nm}$, originating from the release of small molecule volatile matter during carbonization/activation, and mesopores of 10-40 nm (Fig. 3b) arisen by the hydrangea-like surface architecture (tunnels and furrows). A suitable annealing temperature $\left(750{ }^{\circ} \mathrm{C}\right)$ endows the resultant $\mathrm{HHPC}_{750}$ a highest surface area of $2212 \mathrm{~m}^{2} \mathrm{~g}^{-1}$, as shown in Table 1. Lower temperature does not guarantee an entire carbonization/activation of the polymer, and higher one causes a shrink of carbon framework, both giving an obvious drop in surface area. ${ }^{38,}$ 

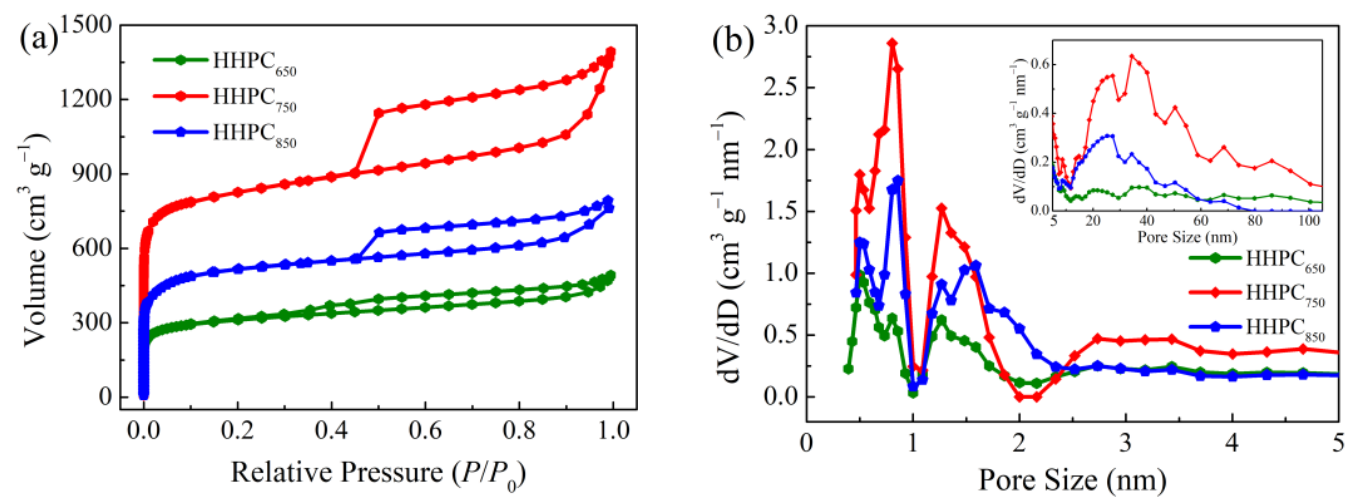

Fig. 3. (a) $\mathrm{N}_{2}$ sorption isotherms and (b) pore size distributions of HHPCs.

Table 1 Surface areas $(S)$, micropore surface areas $\left(S_{\text {micro }}\right)$, pore volumes $\left(V_{\mathrm{t}}\right)$, and chemical compositions of HHPCs.

\begin{tabular}{ccccccc}
\hline Samples & $S\left(\mathrm{~m}^{2} \mathrm{~g}^{-1}\right)$ & $S_{\text {micro }}\left(\mathrm{m}^{2} \mathrm{~g}^{-1}\right)$ & $V_{\mathrm{t}}\left(\mathrm{cm}^{3} \mathrm{~g}^{-1}\right)$ & $\mathrm{C}(\mathrm{wt} \%)$ & $\mathrm{N}(\mathrm{wt} \%)$ & $\mathrm{O}(\mathrm{wt} \%)$ \\
\hline HHPC $_{650}$ & 1167 & 972 & 0.75 & 76.01 & 8.70 & 15.29 \\
HHPC $_{750}$ & 3185 & 2731 & 2.12 & 85.65 & 5.14 & 9.21 \\
HHPC $_{850}$ & 1913 & 1680 & 1.12 & 87.89 & 2.84 & 9.27 \\
\hline
\end{tabular}

The exceptional morphology, pore texture and surface feature of HHPCs inspire us to construct symmetrical CS devices. Two ILs incorporated gel-polymer electrolytes are utilized to investigate the influence of geometric sizes of the constituents on the ion diffusion dynamics within the pores. Solid-state IL/gel-polymer electrolytes can provide the same benefit of the extended voltage window as ILs, and exhibit high competitiveness with conventional liquid-based energy storage systems. Moreover, they are environmentally benign, highly safe, and not easily leaked and flexible. ${ }^{40,41} \mathrm{CV}$ profiles exhibit rectangle-like shapes (Fig. 4a, b) which is indicative of ideal electric double-layer behaviors with excellent charge/discharge properties. ${ }^{42}$ Compared with $\mathrm{HHPC}_{650}$ and $\mathrm{HHPC}_{850}$ electrodes, $\mathrm{HHPC}_{750}$ displays the highest capacitance in $\mathrm{EMIMBF}_{4} / \mathrm{PVDF}_{-}$ HFP and EMIMTFSI/PVDF-HFP electrolytes, which can be attributed to the highest surface area 
and suitable N/O species. As shown in Fig. S5, the lower contact angles of $31-73^{\circ}$ for $\mathrm{EMIMBF}_{4}$ and $21-66^{\circ}$ for EMIMTFSI on HHPC surfaces than those of activated carbon $\left(123 / 122^{\circ}\right)$ manifest enhanced surface wettability due to N/O heteroatom doping. ${ }^{43}$ The carbons with high affinity to electrolytes benefit fast ion transportation into the active surfaces and thereby achieve enhanced ion-accessible surface areas for electric double-lay capacitance. ${ }^{44} \mathrm{CV}$ profiles of $\mathrm{HHPC}_{750} / \mathrm{HHPC}_{750}$ solid-state CS operated in various potential windows (Fig. 4c, d) demonstrate that the device can stably work at $3.5 \mathrm{~V}$ with no obvious increase of anodic current in IL/gel electrolytes. ${ }^{45}$ Symmetric and linear GCD curves (Fig. 4e, f) reflect the excellent capacitive behavior and superb reversibility. ${ }^{46,}{ }^{47} \mathrm{HHPC}_{750}$ electrode in $\mathrm{EMIMBF}_{4} / \mathrm{PVDF}-\mathrm{HFP}$ electrolyte exhibits gravimetric and volumetric capacitances as high as $218 \mathrm{~F} \mathrm{~g}^{-1}$ and $105 \mathrm{~F} \mathrm{~cm}^{-3}$ at $1 \mathrm{~A} \mathrm{~g} \mathrm{~g}^{-1}$, superior to those in EMIMTFSI/PVDF-HFP electrolyte $\left(184 \mathrm{~F} \mathrm{~g}^{-1}\right.$ and $\left.88 \mathrm{~F} \mathrm{~cm}^{-3}\right)$. Furthermore, the electrode capacity remains $167\left(\mathrm{EMIMBF}_{4} / \mathrm{PVDF}-\mathrm{HFP}\right)$ and $118 \mathrm{~F} \mathrm{~g}^{-1}$ (EMIMTFSI/PVDFHFP) at $10 \mathrm{~A} \mathrm{~g}^{-1}$, revealing notable capacitive endurance at large current loads.

Fig. 4g gives Nyquist plots of two $\mathrm{HHPC}_{750}$-based devices, which adopt a fitting equivalent circuit to study the electrolyte ion diffusion and transport kinetics. ${ }^{48}$ In EMIMBF $4 / \mathrm{PVDF}-\mathrm{HFP}$ electrolyte, the device shows lower electrical series resistances $\left(R_{\mathrm{S}}\right.$, the intersections of the real axis) of $2.5 \Omega$ and charge transfer resistances ( $R_{\mathrm{ct}}$, the diameter of semicircle in the high-frequency region) of $4.2 \Omega$, relative to 3.1 and $6.7 \Omega$ in EMIMTFSI/PVDF-HFP. This result manifests a better ion diffusion and charge transfer behavior of EMIMBF 4 -based device. ${ }^{49}$ As displayed in Fig. 4e, a maximum gravimetric energy density of $101.2 \mathrm{Wh} \mathrm{kg}^{-1}$ is achieved at a power density of $875 \mathrm{~W}$ $\mathrm{kg}^{-1}\left(38.7 \mathrm{Wh} \mathrm{L}^{-1}\right.$ at $\left.334 \mathrm{~W} \mathrm{~L}^{-1}\right)$ for the cell assembled with EMIMBF $4 / \mathrm{PVDF}$ HFP electrolyte, which is $29.2 \%$ higher than that of EMIMTFSI/PVDF-HFP device. To our best knowledge, the $\mathrm{HHPC}_{750}$ and $\mathrm{EMIMBF}_{4} / \mathrm{PVDF}-\mathrm{HFP}$ based supercapacitor represents the highest energy density in 
solid-state CSs to date. More impressively, the energy outputs still remain 69.8 and $45.9 \mathrm{Wh} \mathrm{kg}^{-1}$ for $\mathrm{EMIMBF}_{4} / \mathrm{PVDF}-\mathrm{HFP}$ and EMIMTFSI/PVDF-HFP based devices at an ultrahigh power density of $17.5 \mathrm{~kW} \mathrm{~kg}^{-1}$. For a comparison, we also fabricated liquid-based supercapacitors using these two ILs (Fig. S6) which show basically the same energy outputs as the solid-state devices. This result illustrates that the introduction of polymer gel into the IL electrolytes does not obviously affect the electrochemical properties of the resultant CSs, but can address their leakage concerns. The capacitance plots followed by current density increasing and cycle performance within ten cycles of the two devices are exhibited in Fig. S7. After consecutive cycles and high-current tests, the electrode capacitance reversibly recovers to the initial value once the current density goes back to $0.5 \mathrm{~A} \mathrm{~g}^{-1}$, demonstrating prominent electrochemical reversibility and structural robustness of the active materials and the IL/gel-polymer electrolytes. In addition, the both devices exhibit high energy reservation of 91.9 and $90.7 \%$ after 10,000 successive cycles (Fig. 4i), with high Coulombic efficiencies of 99.3 and $98.7 \%$, validating the superior long-term cyclic stability and outstanding reversibility.
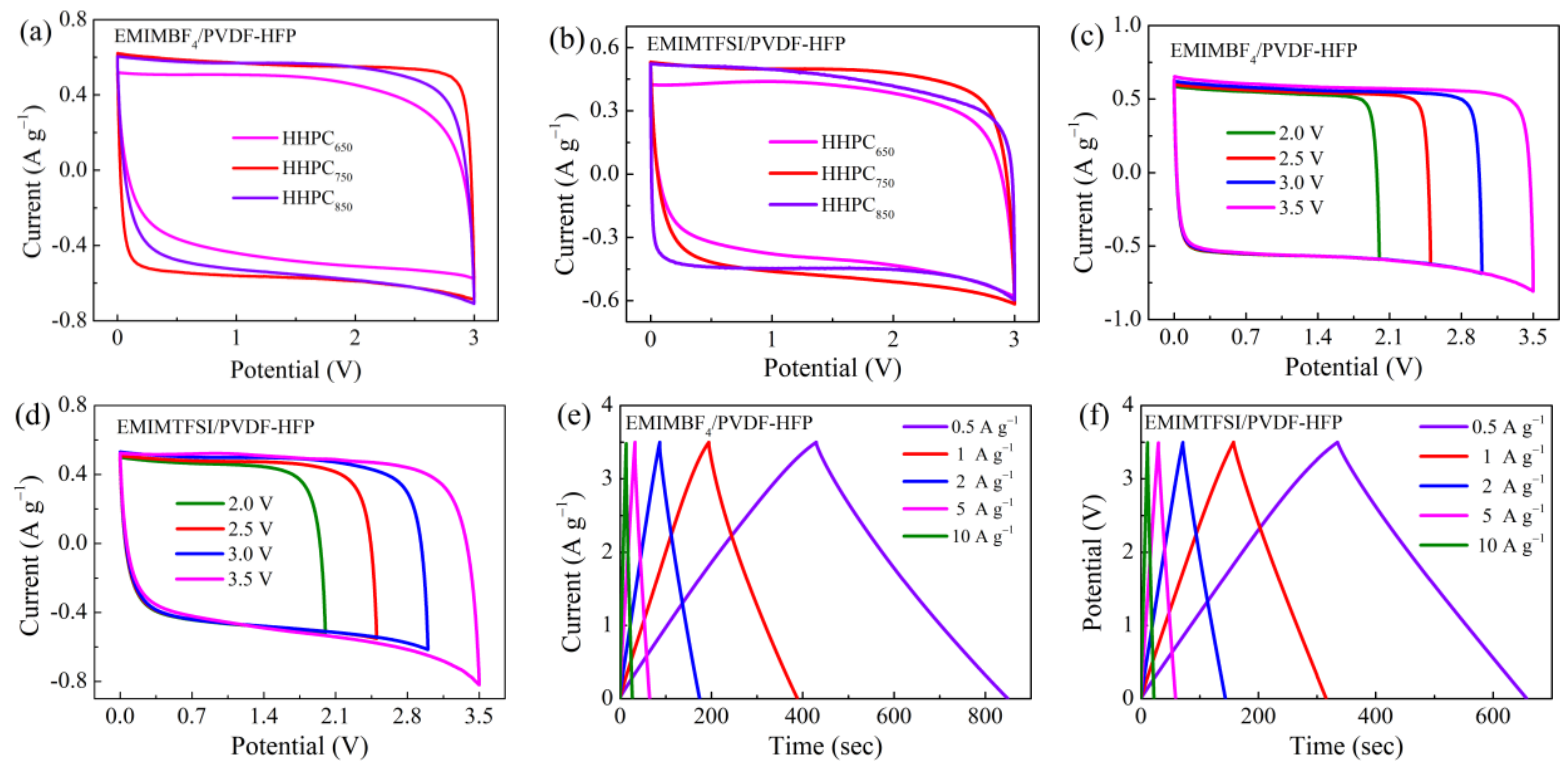

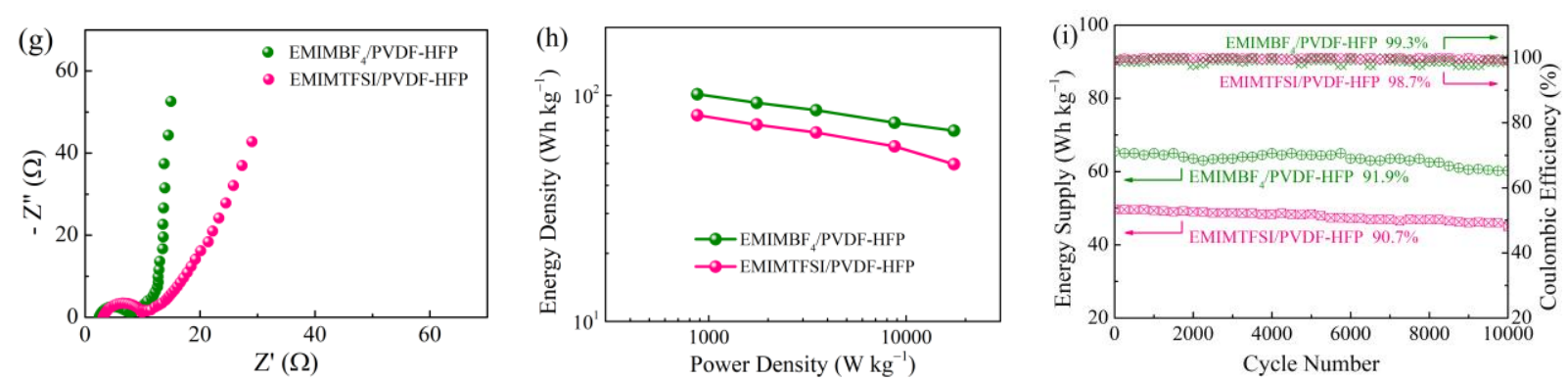

Fig. 4. $\mathrm{CV}$ curves at $10 \mathrm{mV} \mathrm{s}^{-1}$ of HHPCs-based devices employing (a) EMIMBF $4 / \mathrm{PVDF}-\mathrm{HFP}$ and (b) EMIMTFSI/PVDF-HFP electrolyte, respectively; (c, d) CV profiles at different voltage windows, (e, f) GCD curves at various current densities, (g) Nyquist plots; (h) Ragone plots, (i) cycling stability and Coulombic efficiency at $10 \mathrm{Ag}^{-1}$ of the CSs using EMIMBF 4 PVDF-HFP and EMIMTFSI/PVDF-HFP electrolytes.

The temperature-dependent energy densities of EMIMBF $4 / \mathrm{PVDF}-\mathrm{HFP}$ based CS are displayed in Fig. S8. The energy output of the device dramatically increases to $137.6 \mathrm{Wh} \mathrm{kg}^{-1}$ at $90{ }^{\circ} \mathrm{C}, 148 \%$ of that tested at $30{ }^{\circ} \mathrm{C}$. The remarkable boost in energy density can be mainly attributed to that the high temperature lows the electrolyte viscosity, which gives enhanced ionic conductivity and quicker mobility of ions from electrolyte to electrode interface, ${ }^{50,51}$ increasing the surface utilization and hence achieving improved energy storage. Notably, the device energy basically recovers to the initial value when the temperature goes back to $-10{ }^{\circ} \mathrm{C}$. A red light-emitting diode (LED) bulb powered by the solid-state device can normally work with a reliable energy supply under different temperatures, indicating wide applicable operation temperature and thus great potential uses of the device for environmentally adaptable energy supply.

The achievement on high-energy $\mathrm{HHPC}_{750} / / \mathrm{HHPC}_{750}$ solid-state $\mathrm{CS}$ can mainly be ascribed to three aspects: well-designed electrode material, high-voltage IL electrolyte, and the most important point of size matching between both. First, high-surface-area HHPCs with ingenious hydrangealike texture enables sufficient interfaces for charge accumulation, and provides efficient ion 
diffusion kinetics. Meanwhile, N/O species endow the carbon surfaces with good affinity and enhanced wettability, which facilitate electrolyte ions enter and soak into the pore channels and increase ion-accessible surface area. Second, EMIMBF 4 and EMIMTFSI ILs with a wide potential window of $3.5 \mathrm{~V}$ allows the enhancement of the maximum energy supply. Third, the superior electrochemical performances of EMIMBF 4 -based cell benefit most from well-matching between pore architecture and ion dimension (Fig. 5).

The electrode capacitance is closely related to the distance between the ion center and pore wall according to the form of $C=\varepsilon A / d,{ }^{24}$ where $C$ denotes the capacity, $\varepsilon$ represents the dielectric constant of electrolyte, $A$ is the surface area of electrode, and $d$ is the distance between pore surface and ions. If the ionic diameter $\left(d_{\text {ion }}\right)$ is far less than the pore width $(D)$, electrolyte ions positioned around the center of large pores would be at a wide distance $(d)$ to the pore wall, resulting in a capacitance decline. The capacitance has an abnormal promotion when the $D / d_{\text {ion }}$ ratio is in the range of $1.0<$ $D / d_{\text {ion }}<1.7 .{ }^{13,24}$ The ratio of $1.26 \mathrm{~nm}$ micropore in $\operatorname{HHPCs}$ to $\operatorname{EMIM}^{+}(0.76 \mathrm{~nm})$ or TFSI $^{-}(0.79 \mathrm{~nm})$ is just within this range. Moreover, the closer this ratio is to 1 , the larger the capacity will be, ${ }^{52}$ i.e., ion/pore size matching is a key to maximize the electrode capacitance. HHPCs exhibit controlled pore size distribution in which the major subnanopores of $0.80 \mathrm{~nm}$ are perfectly adapted to $\mathrm{EMIM}^{+}$ cations, and the subordinate pores of $0.5 \mathrm{~nm}$ are comparable with $\mathrm{BF}_{4}^{-}$anions $(0.48 \mathrm{~nm})$. This minimizes the free space and guides the oriented arrangement of electrolyte ions along the pore outspread direction, achieving excellent ion transportation pathways and improved ions adsorption number at the pore surface. Nevertheless, the electrolyte ions are difficult to get into the slit pores if the ion size is larger than that of the pores, which prevent the formation of EDLC. Compared with $\mathrm{BF}_{4}^{-}$anions, $\mathrm{TFSI}^{-}$has a larger size which is inaccessible into the $0.5 \mathrm{~nm}$ pores, leading to a lower capacitance and energy density of the fabricated device $\left(101.2 \mathrm{Wh} \mathrm{kg}^{-1}\right.$ for EMIMBF 4 and 
78.3 $\mathrm{Wh} \mathrm{kg}^{-1}$ for EMIMTFSI). Therefore, making full use of the well-designed pores in HHPCs significantly improve the electrochemical energy storage performances.

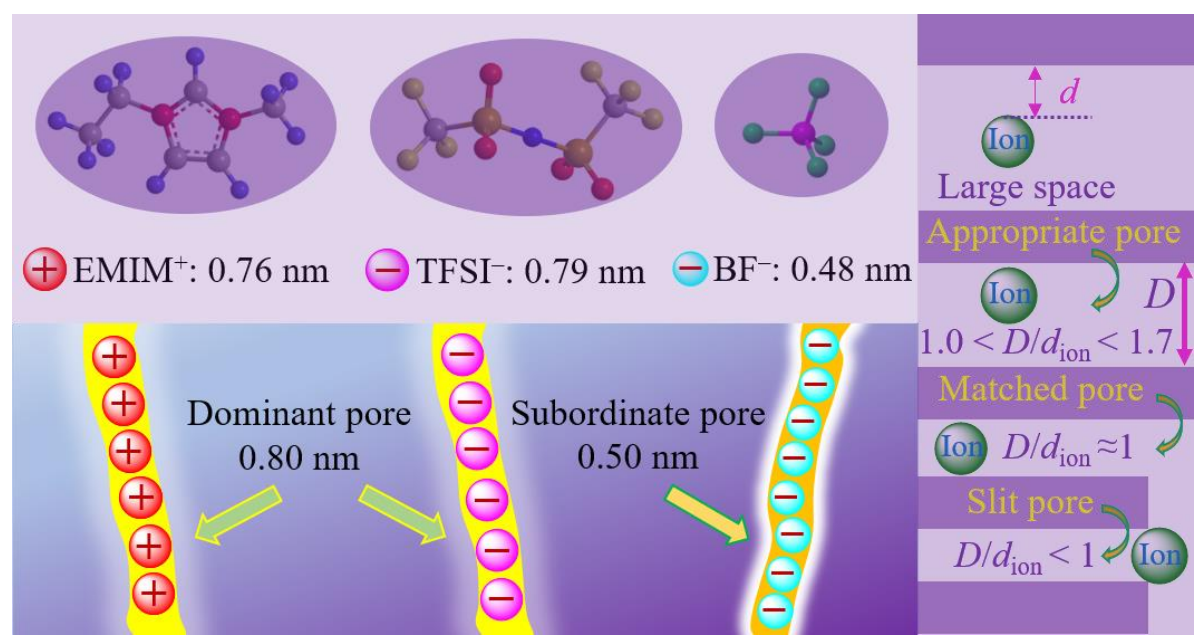

Fig. 5. Schematic illustration of the ion matching in pores with various sizes.

The leakage current and self-discharge behaviors of supercapacitors are a matter of major practical significance in their operation. As shown in Fig. 6a, a small leakage current of $9.7 \mu \mathrm{A}$ can be achieved after $10 \mathrm{~h}$, indicative of less shuttle reactions caused by the impurities in carbons. ${ }^{53}$ Moreover, the self-discharge course undergoes a rapid decline in the beginning several hours and the final output voltage stabilizes at $2.72 \mathrm{~V}$ after $30 \mathrm{~h}$ with $77.7 \%$ reservation of the original charged potential, which is highly desirable for real applications. To satisfy the specific energy and power demands, the operational characteristics of multiple supercapacitors working simultaneously were explored. As displayed in Fig. 6b and c, two devices can be connected to integrate in series to achieve even higher operating voltages and in parallel to obtain larger output currents. The voltage window or discharge time of the device in series or in parallel is doubled compared with a single device. The insets show that two devices in series/parallel can power one green LED, demonstrating superior energy and power supply of the solid-state devices. ${ }^{54}$

To investigate the potential use of our supercapacitor in wearable and portable electronics from a practical point of view, the mechanical flexibility characteristic was performed on the device 
constructed by stacking two symmetric HHPC electrodes with the EMIMBF $4 / \mathrm{PVDF} \mathrm{HFP}$ gelpolymer electrolyte sandwiching between them. There is no obvious distortion in the CV and GCD profiles (Fig. 6d-f), with high retention of capacitance (90.3\%) and Coulombic efficiency (97.5\%) from the initial flat to $180^{\circ}$ bending state. This means the ion/charge transport kinetics are not significantly affected, implying highly flexibility and outstanding electrochemical stability of the device. In addition, the device still holds $87.5 \%$ capacitance reservation even after being bent for 1000 cycles at $180^{\circ}$ (Fig. S9), further confirming the robust mechanical property of the flexible solid-state device.
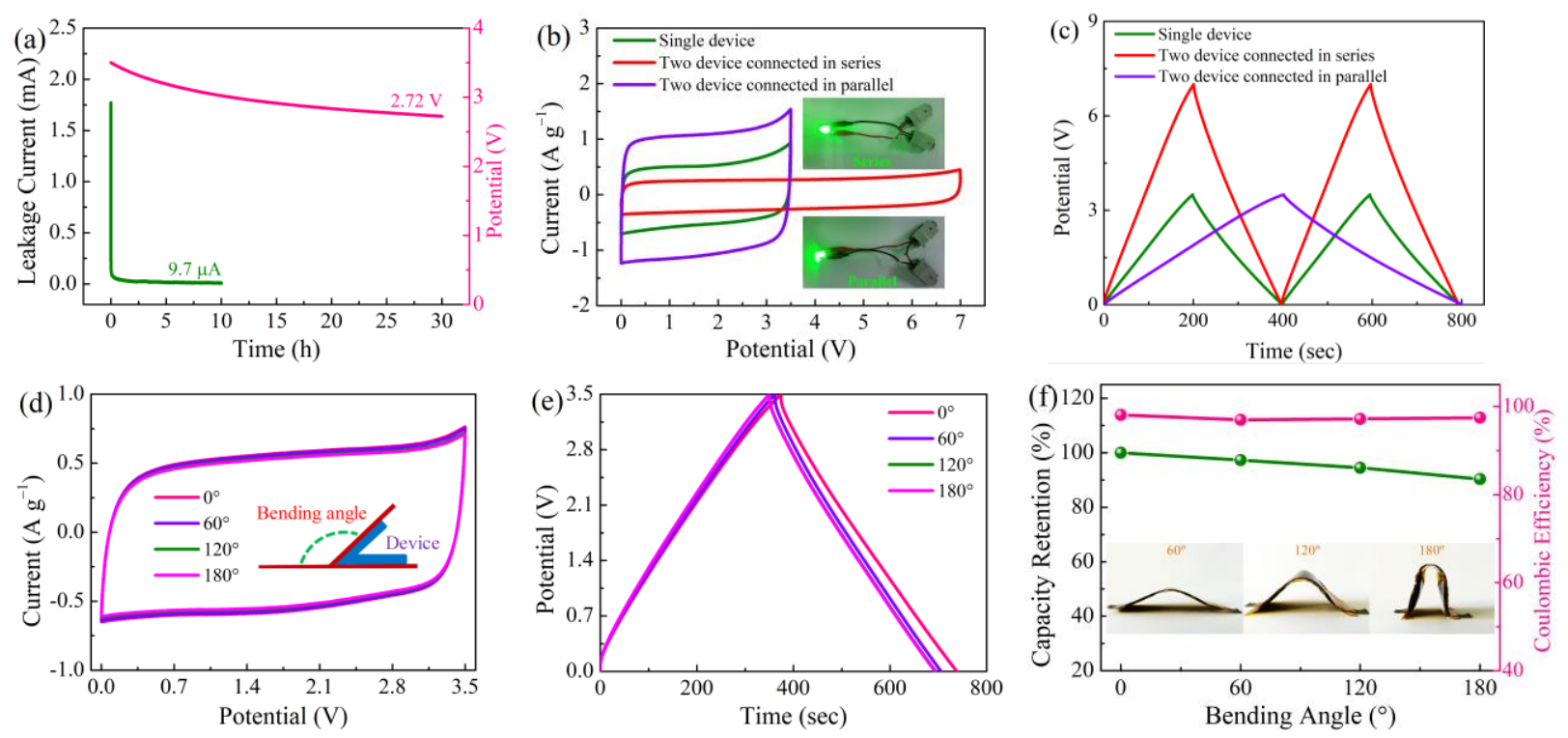

Fig. 6. (a) Leakage current measurement and self-discharge curve of the EMIMBF $4 /$ PVDF-HFP based device; (b) CV profiles and (c) GCD curves of two devices connected in series and parallel; (d) CV profiles, (e) GCD curves, (f) capacity retention as a function of the bending angles (Insets are the photographs of the flexible device bended at $60-180^{\circ}$ ) of the constructed flexible solidstate supercapacitor based on $\mathrm{HHPC}_{750}$ electrodes and EMIMBF $4 / \mathrm{PVDF} \mathrm{HFP}$ electrolyte.

\section{Conclusion}

In conclusion, we exert the pore-ion size matching guideline to engineer HHPCs with exquisitely adapted pore dimension for IL gel electrolytes in the buliding of advanced 
supercapacitors. The exceptional carbon materials are developed based on a facile benzidinebenzoquinone synthetic strategy, which show superior hydrangea-like surface architecture, large surface area, high-level heteroatoms and multiscaled pore network. There is a dramatic differential charge storage in the CSs using two $\mathrm{EMIMBF}_{4}$ and EMIMTFSI gel electrolytes, which originated from the diameters of electrolyte constituents and the consequent ion diffusion and adsorption behaviors within carbon nanopores. The $\mathrm{HHPC}_{750} / \mathrm{HHPC}_{750}$ solid-state supercapacitor using a more matched EMIMBF 4 /PVDF-HFP gel electrolyte delivers a remarkable energy density of 101.2 Wh kg-1 against the replacement of EMIMTFSI (78.3 $\mathrm{Wh} \mathrm{kg}^{-1}$ ). Besides, the constructed solidstate device shows outstanding temperature robustness, excellent series/parallel features, and notably mechanical flexibility. High-capacitance electrode, high-voltage electrolyte, and a key pore-ion size matching between both are crucial to unlock the energy potential of solid-state supercapacitors to a new level. This work demonstrates a break-through in the bottleneck to design functionalized carbons with brilliant narrow ion-comparable pore structure, opening a new door for the development of high-energy solid-state CSs.

\section{Conflicts of interest}

The authors declare no conflict of interest.

\section{Acknowledgements}

This work was financially supported by the National Natural Science Foundation of China (Grant Nos. 21875165, 51772216, 21501135), the Science and Technology of Shanghai Municipality, China (Grant No.14DZ2261100), the Fundamental Research Funds for the Central Universities.

\section{References}


1. Y. Li, S. Zheng, X. Liu, P. Li, L. Sun, R. Yang, S. Wang, Z. S. Wu, X. Bao and W. Q. Deng, Angew. Chem. Int. Ed., 2018, 57, 7992-7996.

2. P. Zhang, J. Wang, W. Sheng, F. Wang, J. Zhang, F. Zhu, X. Zhuang, R. Jordan, O. G. Schmidt and X. Feng, Energy Environ. Sci, 2018, 11, 1717-1722.

3. K. Nomura, H. Nishihara, N. Kobayashi, T. Asada and T. Kyotani, Energy Environ. Science, $2019, \mathbf{1 2}, 1542-1549$.

4. J. Yang, C. Yu, C. Hu, M. Wang, S. Li, H. Huang, K. Bustillo, X. Han, C. Zhao, W. Guo, Z. Zeng, H. Zheng and J. Qiu, Adv. Funct. Mater., 2018, 28, 1803272.

5. P. Zhang, F. Wang, M. Yu, X. Zhuang and X. Feng, Chem. Soc. Rev., 2018, 47, 7426-7451.

6. D. Sheberla, J. C. Bachman, J. S. Elias, C. J. Sun, Y. Shao-Horn and M. Dinca, Nat. Mater., $2017,16,220-224$.

7. T. Lin, I.-W. Chen, F, Liu, C, Yang, H, Bi, F, Xu, F, Huang, Science, 2015, 350, 1508-1513.

8. T. Lv, M. Liu, D. Zhu, L. Gan and T. Chen, Adv. Mater., 2018, 30, 1705489.

9. Z. Zhang, M. Liao, H. Lou, Y. Hu, X. Sun and H. Peng, Adv. Mater., 2018, 30, 1704261.

10. Q. Dou, S. Lei, D.-W. Wang, Q. Zhang, D. Xiao, H. Guo, A. Wang, H. Yang, Y. Li, S. Shi and X. Yan, Energy Environ. Sci., 2018, 11, 3212-3219.

11. N. Zhang, F. Liu, S.-D. Xu, F.-Y. Wang, Q. Yu and L. Liu, J. Mater. Chem. A, 2017, 5, $22631-22640$.

12. Z. Ling, Z. Wang, M. Zhang, C. Yu, G. Wang, Y. Dong, S. Liu, Y. Wang and J. Qiu, Adv. Funct. Mater, 2016, 26, 111-119.

13. X. Wang, H. Zhou, E. Sheridan, J. C. Walmsley, D. Ren and D. Chen, Energy Environ. Sci., $2016,9,232-239$.

14. P. Zhang, Y. Li, G. Wang, F. Wang, S. Yang, F. Zhu, X. Zhuang, O. G. Schmidt and X. Feng, 
Adv. Mater., 2019, 31, 1806005.

15. H. Peng, G. Qian, N. Li, Y. Yao, T. Lv, S. Cao and T. Chen, Advanced Science, 2018, 1800784.

16. K. L. Van Aken, M. Beidaghi and Y. Gogotsi, Angew. Chem. Int. Ed., 2015, 54, 4806-4809.

17. X. Wang, A. Y. Mehandzhiyski, B. Arstad, K. L. Van Aken, T. S. Mathis, A. Gallegos, Z. Tian, D. Ren, E. Sheridan, B. A. Grimes, D. E. Jiang, J. Wu, Y. Gogotsi and D. Chen, J. Am. Chem. Soc., 2017, 139, 18681-18687.

18. P. Pachfule, D. Shinde, M. Majumder and Q. Xu, Nat. Chem., 2016, 8, 718-724.

19. W. Guo, C. Yu, S. Li, X. Song, H. Huang, X. Han, Z. Wang, Z. Liu, J. Yu, X. Tan and J. Qiu, Adv. Mater., 2019, 1901241.

20. X. Zhuang, F. Zhang, D. Wu and X. Feng, Adv. Mater., 2014, 26, 3081-3086.

21. J. Zhao, Y. Li, G. Wang, T. Wei, Z. Liu, K. Cheng, K. Ye, K. Zhu, D. Cao and Z. Fan, J. Mater. Chem. A, 2017, 5, 23085-23093.

22. L. Estevez, V. Prabhakaran, A. L. Garcia, Y. Shin, J. Tao, A. M. Schwarz, J. Darsell, P. Bhattacharya, V. Shutthanandan and J. G. Zhang, ACS Nano, 2017, 11, 11047-11055.

23. G. Y. J. Chmiola, Y. Gogotsi, C. Portet, P. Simon, P. L. Taberna, Science, 2006, 313, 1761.

24. C. Largeot, C. Portet, J. Chmiola, P.-L. Taberna, Y. Gogotsi, and P. Simon, J. Am. Chem. Soc., 2008, 130, 2730-2731.

25. C. Lian, D.-e. Jiang, H. Liu and J. Wu, The J. Phys. Chem. C, 2016, 120, 8704-8710.

26. H. Liu, G. Zhang, Y. Zhou, M. Gao and F. Yang, J. Mater. Chem. A, 2013, 1, 13902.

27. S. Zhang, J. Zhu, Y. Qing, L. Wang, J. Zhao, J. Li, W. Tian, D. Jia and Z. Fan, Adv. Funct. Mater., 2018, 28, 1805898.

28. D. Zhu, J. Jiang, D. Sun, X. Qian, Y. Wang, L. Li, Z. Wang, X. Chai, L. Gan and M. Liu, J. 
Mater. Chem. A, 2018, 6, 12334-12343.

29. G. Zhao, C. Chen, D. Yu, L. Sun, C. Yang, H. Zhang, Y. Sun, F. Besenbacher and M. Yu, Nano Energy, 2018, 47, 547-555.

30. C. Hu, D. Creaser, S. Siahrostami, H. Grönbeck, H. Ojagh and M. Skoglundh, Catal. Sci. Technol., 2014, 4, 2427-2444.

31. L. Ma, J. Liu, S. Lv, Q. Zhou, X. Shen, S. Mo and H. Tong, J. Mater. Chem. A, 2019, 7, $7591-7603$.

32. T. Wang, Y. Sun, L. Zhang, K. Li, Y. Yi, S. Song, M. Li, Z. A. Qiao and S. Dai, Adv. Mater, $2019,1807876$.

33. L.-F. Chen, Y. Lu, L. Yu and X. W. Lou, Energy Environ. Sci., 2017, 10, 1777-1783.

34. Y. Li, G. Wang, T. Wei, Z. Fan and P. Yan, Nano Energy, 2016, 19, 165-175.

35. D. Hulicova-Jurcakova, M. Seredych, G. Q. Lu and T. J. Bandosz, Adv. Funct. Mater., 2009, 19, $438-447$.

36. Y. Liu, Z. Xiao, Y. Liu and L.-Z. Fan, J. Mater. Chem. A, 2018, 6, 160-166.

37. Y. Liu, X. Qiu, X. Liu, Y. Liu and L.-Z. Fan, J. Mater. Chem. A, 2018, 6, 8750-8756.

38. J.-S. M. Lee, M. E. Briggs, C.-C. Hu and A. I. Cooper, Nano Energy, 2018, 46, 277-289.

39. G. Shen, X. Sun, H. Zhang, Y. Liu, J. Zhang, A. Meka, L. Zhou and C. Yu, J. Mater. Chem. $A, 2015,3,24041-24048$.

40. H. Li, Y. Hou, F. Wang, M. R. Lohe, X. Zhuang, L. Niu, and X. Feng, Adv. Energy Mater., $2017,7,1601847$.

41. H. Li, T. Lv, H. Sun, G. Qian, N. Li, Y. Yao and T. Chen, Nat. Commun., 2019, 10, 536.

42. F. Wei, X. He, H. Zhang, Z. Liu, N. Xiao and J. Qiu, J. Power Sources, 2019, 428, 8-12.

43. T. Ouyang, K. Cheng, Y, Gao, S, Kong, K, Ye, G, Wang and D, Cao, J. Mater. Chem. A, 
2016, 4, 9832-9843.

44. D. Xue, D. Zhu, W. Xiong, T. Cao, Z. Wang, Y. Lv, L. Li, M. Liu and L. Gan, ACS Sustainable Chem. Eng., 2019, 7, 7024-7034.

45. K. Lu, B. Song, K. Li, J. Zhang and H. Ma, J. Power Sources, 2017, 370, 98-105.

46. X. He, X. Xie, J. Wang, X. Ma, Y. Xie, J. Gu, N. Xiao and J. Qiu, Nanoscale, 2019, 11, $6610-6619$.

47. L. Miao, X. Qian, D. Zhu, T. Chen, G. Ping, Y. Lv, W. Xiong, Y. Liu, L. Gan and M. Liu, Chin. Chem. Lett., 2019, 30, 1445-1449.

48. Z. Song, H. Duan, L. Li, D. Zhu, T. Cao, Y. Lv, W. Xiong, Z. Wang, M. Liu and L. Gan, Chem. Eng. J., 2019, 372, 1216-1225.

49. L. Wang, J. Ding, S. Sun, B. Zhang, X. Tian, J. Zhu, S. Song, B. Liu, X. Zhuang and Yu Chen, Adv. Mater. Interfaces, 2018, 5, 1701679.

50. M. P. Scott, M. Rahman and C. S. Brazel, Eur. Polym. J., 2003, 39, 1947-1953.

51. Z. Song, H. Duan, D. Zhu, Y. Lv, W. Xiong, T. Cao, L. Li, M. Liu and L. Gan, J. Mater. Chem. A, 2019, 7, 15801-15811.

52. C. P. Celine Largeot, John Chmiola, Pierre-Louis Taberna, Yury Gogotsi, and Patrice Simon, J. Am. Chem. Soc., 2008, 130, 2730-2731

53. R. B. K. Maher F. El-Kady, Nat. Commun., 2013, 4, 1475.

54. Z. Pan, J. Yang, Q. Zhang, M. Liu, Y. Hu, Z. Kou, N. Liu, X. Yang, X. Ding, H. Chen, J. Li, K. Zhang, Y. Qiu, Q. Li, J. Wang and Y. Zhang, Adv. Energy Mater., 2019, 9, 1802753. 